\title{
Gekommen und geblieben
}

\section{Matthias Scholer}

Redaktor Online und Print

Rund ein Drittel der Ärztinnen und Ärzte in der Schweiz kommen aus dem Ausland. Weshalb haben sie sich für ein Leben hierzulande entschieden? Welches sind kulturelle Stolperfallen, die es zu umschiffen gilt? Und was halten sie von der Schweizer Gesundheitspolitik? Diesen und weiteren Fragen möchten wir in der neuen, in loser Folge erscheinenden Artikelserie "Grüezi Schweiz» nachgehen. Den Anfang macht das Porträt der Hausärztin Kathleen Keilwerth, die eigentlich gar nie in die Schweiz kommen wollte.

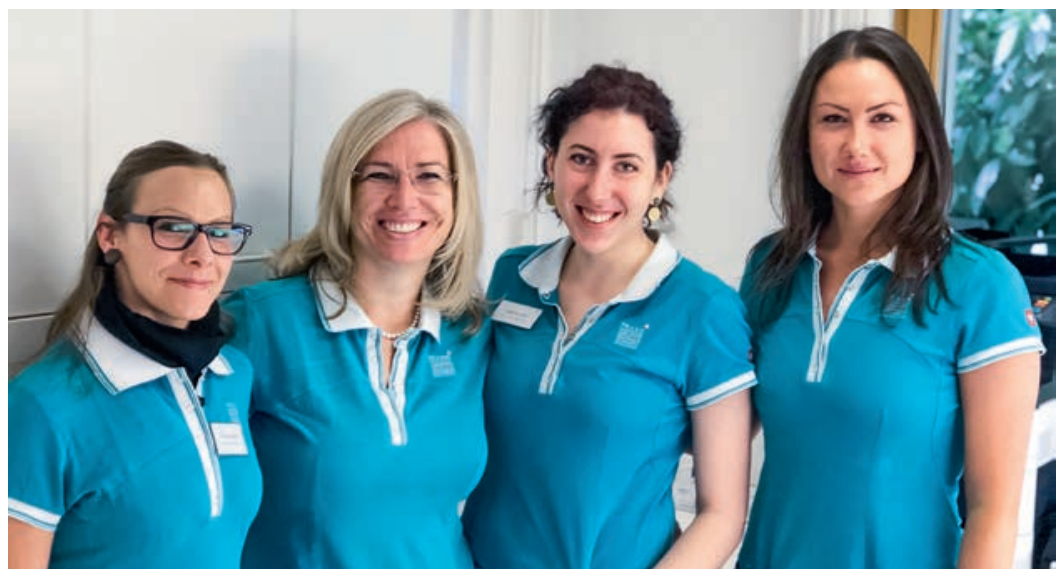

Kathleen Keilwerth (Zweite von links) mit ihrem Team.

Der Weg in die Schweiz

Erstens kommt es anders, zweitens als man denkt. Eine Redensart, die definitiv auf den Werdegang von Kathleen Keilwerth zutrifft. Ursprünglich wollte die gebürtige Ostdeutsche als Rechtsmedizinerin oder Chirurgin arbeiten. «Für mein Wahlstudienjahr wählte ich jedoch Psychiatrie als Fachrichtung», erzählt die Ärztin. Deshalb bewarb sie sich als Unterassistentin auf eine freie Stelle in der Klinik Waldhaus in Chur und wurde prompt dafür ausgewählt. «Ich nahm die Stelle mit gemischten Gefühlen an, denn eigentlich wollte ich gar nicht in die Schweiz", erinnert sich Keilwerth. Im Nachhinein entpuppte sich die Stelle jedoch in mehrfacher Hinsicht als Gewinn. «In Deutschland werden den Unterassistenten normalerweise die doofsten Aufgaben zugeteilt», so Kathleen Keilwerth. Ganz anders in Chur. Hier durfte sie als Studentin nicht nur mit auf Oberarztvisite. Ihr wurden auch verantwortungsvolle Aufgaben übertragen. Doch auf
Keilwerth wartete noch der Jackpot: «Kurz vor dem Ende meiner Zeit in Chur verliebte ich mich.» Dieser Liebe wegen zog Kathleen Keilwerth nach dem Staatsexamen an der Universität Ulm zurück nach Graubünden. Hier absolvierte sie ihre Assistenzzeit und arbeitete danach als Oberärztin im Landesspital Vaduz auf der Inneren Medizin. Auch privat ging es vorwärts. Kathleen Keilwerth heiratete und wurde Mutter eines Jungen.

Wenige Jahre später kam es zu einer jähen Zäsur: Ihr Mann starb. «Damit änderten sich auch in beruflicher Hinsicht die Prioritäten», erzählt die Ärztin. Keilwerth arbeitete zuerst in einer Notfallpraxis. Seit letztem Dezember führt sie nun in Untervaz im Angestelltenverhältnis eine Hausarztpraxis. Bereut sie nicht, nun als Hausärztin und nicht wie ursprünglich geplant als Chirurgin oder Rechtsmedizinerin zu arbeiten? «Definitiv nicht. Ich bin fest davon überzeugt, dass der Hausarztberuf meine Berufung ist.»

\section{Vorsichtige Annäherung und angedrohtes Kartoffelstockverbot}

Keilwerths Vorgänger suchte während Jahren vergeblich jemanden, der seine Praxis übernehmen wollte. «Entsprechend froh waren seine Patienten, als er mich als seine Nachfolgerin vorstellte», erzählt Keilwerth.

Der Hausärztin kam immer wieder mal zu Ohren, dass sich Patienten vorgängig bei Berufskollegen erkundigten, ob man der Deutschen denn trauen könne.

Ein Vorteil sei sicher auch gewesen, dass sie bereits während vierzehn Jahren in Chur lebte und entsprechend vernetzt war. Trotzdem kam es der Hausärztin 
immer wieder mal zu Ohren, dass sich Patienten vorgängig bei Berufskollegen erkundigten, ob man der Deutschen denn trauen könne. Diese Skepsis hat sich jedoch rasch gelegt. "Heute bin ich eine Person des öffentlichen Lebens. Die Leute grüssen mich auf der Strasse und ich bekomme regelmässig nette Rückmeldungen», freut sich Kathleen Keilwerth. Stellt der Bündner Dialekt keine Hürde dar? «Also mit dem Verstehen habe ich kein Problem. Aber ich habe es aufgegeben, selber Dialekt sprechen zu wollen", erzählt die Ärztin lachend. Vor allem die Aussprache des Wortes "Herdöpfelstogg» sei für sie nicht zu meistern. Ihre MPAs haben ihr sogar gedroht, dass sie erst wieder Kartoffelstock essen darf, wenn sie das Wort fehlerfrei wiedergeben könne.

\section{Die Schweiz, ein Traumarbeitsort für Ärztinnen und Ärzte?}

"Jein», sagt Kathleen Keilwerth. Ein häufiger Fehler, den in ihren Augen viele Deutsche machen: Sie denken, die Schweiz sei ihrem Heimatland sehr ähnlich. «Die Eidgenossenschaft ist jedoch ein Land mit eigenen Regeln und einer völlig unterschiedlichen Mentalität», gibt Keilwerth zu bedenken. Es sei nicht einfach, hierzulande Fuss zu fassen. Und eine latente Aversion gegenüber den Deutschen sei nicht wegzudiskutieren. «Die Schweizer sind nicht so niedlich, wie sie in der RicolaWerbung dargestellt werden", ergänzt Kathleen Keilwerth lachend. Deshalb rät die Ärztin denn auch ihren Kolleginnen und Kollegen, sich den Schritt, in die Schweiz umzuziehen, gut zu überlegen. Sogar einen interkulturellen Workshop bei Arbeitsbeginn würde sie empfehlen - damit könnten einige vorhersehbare Konflikte vermieden werden.

\section{Kulturelle Stolperfallen}

Doch welches sind denn die grössten Stolperfallen, in die deutsche Kolleginnen und Kollegen gerne mal tappen? «Der forsche Ton der Deutschen führt schnell mal zu Unmut bei den Schweizern", weiss Kathleen Keilwerth aus eigener Erfahrung. Und: «Deutsche drängen sich gerne in den Vordergrund. Das kommt hier gar nicht gut an.» Dieser Punkt erinnert sie an eine Anekdote: "Als in Vaduz die Stelle einer Oberärztin frei wurde, kam mein damaliger Chef zu mir und fragte, ob ich an der Stelle interessiert wäre.» In Deutschland würde so etwas komplett anders ablaufen. Da würden sich sofort Dutzende bewerben, und ihnen wäre jedes Mittel recht, um den eigenen Namen ins Spiel und ins Gespräch zu bringen. Ganz anders hierzulande: «Der Schweizer wartet zuerst einmal ab und fragt dann

"Heute ertappe ich mich ab und zu selber, dass mich die etwas gar forsche Art gewisser deutscher Kolleginnen und Kollegen nervt.»

gezielt einzelne Kandidaten an.» Diese Zurückhaltung habe sie anfänglich auch lernen müssen, aber zwischenzeitlich verinnerlicht: "Heute ertappe ich mich ab und zu selber, dass mich die etwas gar forsche Art gewisser deutscher Kolleginnen und Kollegen nervt.»

\section{Was die Schweiz von Deutschland lernen sollte} Vor allem die Diskussionen rund um die Einführung von DRGs im ambulanten Bereich verfolgt Keilwerth mit grossem Interesse. Und hier hat sie eine klare Meinung: «Die Einführung von Fallpauschalen im ambulanten Bereich sollte vermieden werden.» Ihre deutschen Kolleginnen und Kollegen berichten ihr immer wieder, wie nachteilig sich Fallpauschalen für Patienten und Hausärzte auswirken. «In Deutschland erhält man pro Patient 32 Euro pro Quartal. Egal wie häufig man diesen sieht», weiss Kathleen Keilwerth. Massenabfertigung statt persönlicher Patientenbetreuung ist die Folge. «Wenn ich in Deutschland erzähle, was ich für meine Patienten tue, dann glaubt mir das fast niemand. Dabei sind doch die Patientinnen und Patienten Kunden mit einem Serviceanspruch, den es zu erfüllen gilt», ist die Hausärztin überzeugt. Und sie ergänzt: «Gesundheitspolitik sollte nicht auf dem Rücken der Patienten ausgetragen werden.»

\footnotetext{
En Suisse, un tiers environ des médecins vient de l'étranger. Pourquoi ont-ils choisi de vivre ici? Quels sont les pièges culturels qu'ils doivent éviter? Et que pensent-ils de la politique de santé suisse? Nous aimerions répondre à ces questions et à d'autres dans notre nouvelle série d'articles publiés occasionnellement, «Bonjour la Suisse!».

Pour celle-ci, nous sommes à la recherche de médecins étrangers prêts à nous donner un aperçu de leur vie et de leur quotidien professionnel. Nous nous réjouissons de votre prise de contact à l'adresse mscholer[at]emh.ch.
} 